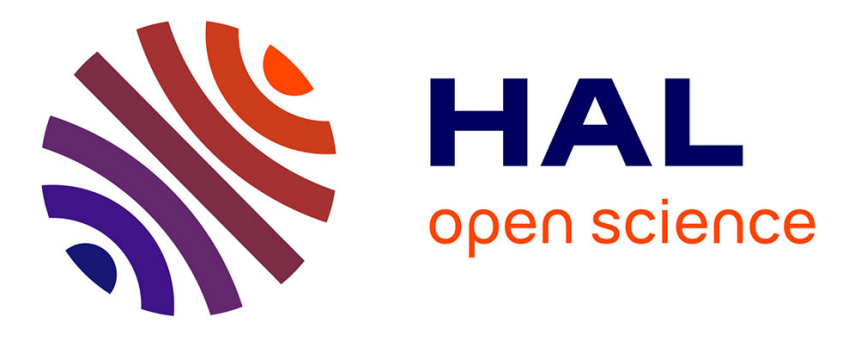

\title{
Regional Sensitivity Analysis Applied To Train Traffic Rescheduling in Case of Power Shortage
}

Soha Saad, Florence Ossart, Jean Bigeon, Etienne Sourdille, Harold Gance

\section{To cite this version:}

Soha Saad, Florence Ossart, Jean Bigeon, Etienne Sourdille, Harold Gance. Regional Sensitivity Analysis Applied To Train Traffic Rescheduling in Case of Power Shortage. IEEE - 13th System of Systems Engineering Conference (SoSE), Jun 2018, Paris, France. pp.459-463, 10.1109/sysose.2018.8428703 . hal-01801271

\section{HAL Id: hal-01801271 \\ https://hal-centralesupelec.archives-ouvertes.fr/hal-01801271}

Submitted on 11 Mar 2020

HAL is a multi-disciplinary open access archive for the deposit and dissemination of scientific research documents, whether they are published or not. The documents may come from teaching and research institutions in France or abroad, or from public or private research centers.
L'archive ouverte pluridisciplinaire HAL, est destinée au dépôt et à la diffusion de documents scientifiques de niveau recherche, publiés ou non, émanant des établissements d'enseignement et de recherche français ou étrangers, des laboratoires publics ou privés. 


\title{
Regional Sensitivity Analysis Applied To Train Traffic Rescheduling in Case of Power Shortage
}

\author{
Soha SAAD ${ }^{(1,2,3)}$, Florence OSSART ${ }^{(1)}$, Jean BIGEON ${ }^{(2)}$, Etienne SOURDILLE ${ }^{(3)}$, Harold GANCE ${ }^{(3)}$ \\ (1) GeePs - CNRS UMR 8507 - CentraleSupélec - UPSud - Sorbonne Université UPMC; 91192 Gif sur Yvette - France \\ (2) G-SCOP - CNRS UMR 5272 ; Grenoble INP-UJF ; 38000 Grenoble - France \\ (3) SNCF Réseaux, Direction de l’ingénierie, 6 avenue Francois Mitterand, 95574, La plaine St-Denis, France
}

\begin{abstract}
The present work addresses train traffic rescheduling that is needed when an electric incident limits the power available for train traction. This is a difficult process of prime importance and we propose a decision-support tool to help the operator. The railway network is a complex multi-physics dynamic system, with many operational constraints, and its simulation is expensive. This makes the management of an incident difficult. The proposed approached applies regional sensitivity in order to study the influence of the different adjustment variables (train delays, speed references ...) on traffic quality indicators and operational constraints (catenary voltage, for example). The analysis is divided in two parts: Monte Carlo filtering for factor mapping (qualitative analysis), and two samples Kolmogorov-Smirnov test for prioritization and fixing factors (quantitative analysis). The results provide information about the behavior of the system and the influence of the different traffic adjustment variables, and help reorganizing the train traffic in an optimal way. As a result of the analysis, we obtain a set of feasible solutions that are organized according to different performance criteria. Pareto-optimal front are plotted in order to guide the decision-maker. The proposed approach is illustrated for a simple case representative of suburban traffic.
\end{abstract}

Keywords - electric railway infrastructure, train traffic rescheduling, multi-criteria decision-making, regional sensitivity analysis, Monte Carlo filtering, Kolgoromov-Smirnov test.

\section{INTRODUCTION}

Developing better solutions for train rescheduling problems has been drawing the attention of researchers for decades. Many research studies in this area deal with situations where a minor incident on a track affects a large number of trains due to cascade effects [1], [2], [3], while others discuss the impact of major incidents [4], [5], [6]. All these studies focus on the availability of rolling stock and crews, but do not take into account electrical infrastructure limits and the issue of electric power availability is never dealt with.

The present work addresses train traffic rescheduling, in the case of electric power shortage, due to an electric equipment outage. Such incidents limit the power available for train traction, and hence the transportation capacity of the railway system. We propose a method to reschedule the train traffic, and to optimally share the available power among the different trains. Our approach is based on regional sensitivity analysis. We use of Monte Carlo filtering and cumulative distribution functions in order to rank the influence of the decision variables while accounting for various operational constraints. The paper is organized as follows: Section 2 describes the problem with more details; Section 3 introduces regional sensitivity analysis and presents how the method is applied to traffic rescheduling; Section 4 provides first results in a simple case and Section 5 concludes.

\section{PRoBlem STATEMENT}

The electrical infrastructure of a railway network is designed and controlled so as to provide the power needed by the trains. It is a complex system, in which the main elements are the feeding sub-stations, the catenaries, the rails (return conductor) and the trains. Other devices allow to configure the electrical network's topology according to the needs. Numerous trains travel at the same time on different lines, and the analysis of the system relies on simulation.

In the present work, we use ESMERALDA NG [7], a simulator developed by the SNCF. This simulator is based on a multiphysical model of the railway network: mechanical, electrical, and thermal. The input data are the physical description of the railway network on one hand (topology, position and characteristics of all devices, including the trains) and the description of the intended traffic on the other hand (type of trains, departure and passage times at various points, reference speed profiles along the way). The equations of train dynamics are coupled with the circuit equations of the electrical network and solved step by step over time in order to determine the position of the different trains at each time, as well as different electrical quantities such as the voltage at the pantographs, powers passing through catenaries and transformers and the resulting heating. It is a nonlinear numerical model with a large number of inputs and outputs. The computation cost is high, with a large number of variables involved and simulation times around ten minutes.

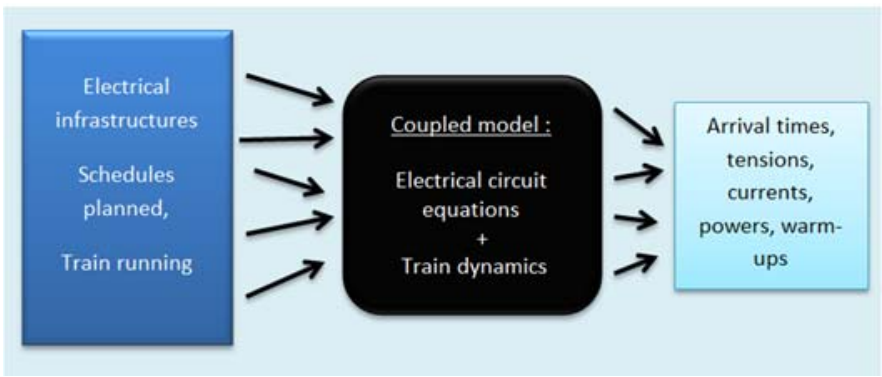

Figure 1: Structure of simulation model

The simulator calculates the actual traffic, for a given physical infrastructure and traffic instructions. For example, a train is scheduled to leave at a certain time, and travel according to a 
certain speed profile, but if the actual available power is too low, the actual speed profile will not be the expected one and the train will be late. From a technical point of view, the quality of power distribution is monitored through the catenary voltage: too small values indicate that the electrical network is overloaded, which is not desired and requires the traffic to be adapted.

If a component of the electrical infrastructure is unavailable, due to either a technical incident or a maintenance operation, the power available for traction is reduced. It is then necessary to check if the residual capacity allows to maintain the traffic initially planned. The criterion is the catenary voltage, which must remain within the range defined by the standards. If not, the train traffic must be rescheduled.

In the current situation, traffic rescheduling is carried out according to an iterative trial-and-errors method: on the basis of their experience, operators propose re-planning solutions and run simulation to check if the catenary voltage remains within the prescribed limits. This process is slow because the analyzed situation is complex (many trains, many tracks) and numerous simulation runs are needed. Furthermore, the outcome of the process, both in terms of quality of the solution and time to reach it, fully depends on the operator experience and know-how. There is no guarantee that an optimal solution will be reached.

The goal of the presented work is to assist the operator in this decision making process, using a tool which manages the simulator and efficiently explores the solution space in order to propose a set of acceptable solutions. 'Acceptable solutions' means traffic grids which respect all the operational constraints of the system. The tool should also provide sensitivity indicators with respect to the different traffic grid parameters (decision variables), and Pareto-optimal front according to criteria defined by the decision maker, in order to guide him for the choice of the final solution.

The proposed approach is based on sensitivity analysis, which is "an integral part" of any decision-making process accompanied by the creation of a decision-support model [8]. Sensitivity analysis has been introduced into the world of decision-making for a long time, especially in the framework of multi-criteria decision making [9], [10], [11], [12]. Sensitivity analysis offers a better understanding of the problem and answers the following questions. Which adjustment operation is the most influential? How does a given input variable affect the output? Which adjustment operators are required to reschedule the traffic and respect operational constraints? Among acceptable solutions, which one are optimal with respect to given criteria such as: maximizing the density of rail traffic, minimizing train departure delays, minimizing train travel time, minimizing train arrival delays?

\section{SENSITIVITY ANALYSIS APPLIED TO TRAFFIC RESCHEDULING}

\subsection{Traffic rescheduling process}

Traffic rescheduling requires to adjust the traffic grid according to the actual power capacity of the railway system: less train can circulate, and/or their speed must be reduced. To do this, a set of decision variables is defined, such as time and/or space intervals between trains or speed limits at particular points of the lines. Each point of the decision variables space corresponds to a particular traffic grid. The decision maker needs to explore this space in order to find points corresponding to acceptable solutions, in the sense that they respect all physical and operational constraints of the system. This process is based on a numerical model which is complex, nonlinear, time consuming, and used as a black box. Conventional operations research methods are not suitable, and we have chosen to use regional sensitivity analysis to better understand the relationships between the inputs and outputs of our model, while accounting for operational constraints.

\subsection{Purposes of sensitivity analysis}

Let us denote $X_{i}$, the inputs of the model to be analyzed. Global sensitivity analysis (GSA) is the process of varying the inputs of the model over their whole range, and observe the resulting changes of the outputs. It explores how changes of the model output can be qualitatively and quantitatively attributed to different model inputs. Usually, three settings are defined [9]:

- Factor Prioritization aims at ranking the inputs in terms of their relative contribution to output variation.

- Factor fixing aims at determining the inputs, if any, that do not have any contribution to output variation.

- Factor mapping aims at determining which part of the inputs space produces specific output values, for instance above a prescribed threshold.

\subsection{Regional sensitivity analysis}

Regional sensitivity analysis (RSA) is used for factor mapping : one is interested in determining what input values cause the model output to be in a certain region, defined by a performance indicator or a constraint. In our case, we want the catenary voltage to be above a given threshold for all trains and at all times. A simple qualitative method is Monte Carlo filtering: Monte Carlo runs are performed and the sampled input space is partitioned into two groups, depending on whether the associated model output satisfies or not the desired condition. The so-called «behavioral group ", denoted by $\left(X_{i} / R\right)$, of size $n$, contains the elements that respect the performance indicators, while the "non-behavioral group ", denoted by $\left(X_{i} / \bar{R}\right)$, of size $\bar{n}$, contains those that do not. The sum $n+\bar{n}$ corresponds to the total number of runs. For each input, the empirical cumulative distribution functions of both groups, respectively denoted by $F_{n}\left(X_{i} / R\right)$ and $F_{\bar{n}}\left(X_{i} / \bar{R}\right)$, are computed and plotted (Fig. 2).

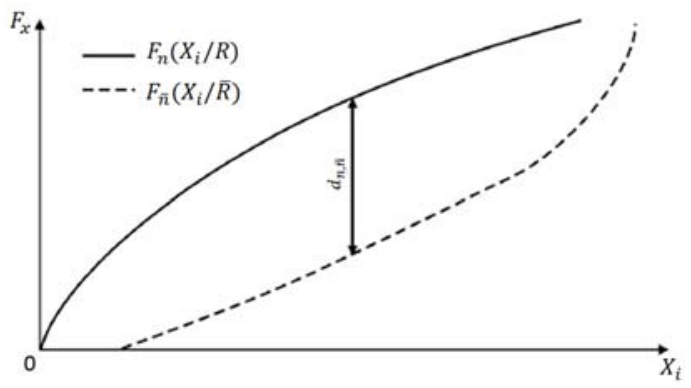

Figure 2: CDF curves of the two sets of values for input variable $X_{i}$

Visual comparison between $F_{n}\left(X_{i} / R\right)$ and $F_{\bar{n}}\left(X_{i} / \bar{R}\right)$ provides qualitative information about the influence of each input variable with respect to factor mapping. The underlying assumption is that if $F_{n}\left(X_{i} / R\right)$ and $F_{\bar{n}}\left(X_{i} / \bar{R}\right)$ are significantly different, then the sensitivity of the performance indicator to $X_{i}$ is high. The shape and the relative positions of the curves also contain information. If $F_{n}\left(X_{i} / R\right)$ lies above $F_{\bar{n}}\left(X_{i} / \bar{R}\right)$, it means that the performance indicator is statistically more respected for smaller values of $X_{i}$ : increasing $X_{i}$ has a negative impact on the indicator performance criterion. Conversely, if $F_{n}\left(X_{i} / R\right)$ lies under $F_{\bar{n}}\left(X_{i} / \bar{R}\right)$, increasing $X_{i}$ has a positive impact. The shape of the curves shows the range of values for which $X_{i}$ is influential. 


\subsection{Two sample Kolmogorov-Smirnov test}

The two-sample Kolmogorov-Smirnov (K-S) test is used to decide if two data sets come from the same continuous distribution or not. In the context of RSA, it provides two quantitative indicators that allow factor prioritization and factor fixing.

The test is applied to the cumulative distribution functions $F_{n}\left(X_{i} / R\right)$ and $F_{\bar{n}}\left(X_{i} / \bar{R}\right)$. The null hypothesis $H_{0}$ is that the two functions are identical, $F_{n}\left(X_{i} / R\right)=F_{\bar{n}}\left(X_{i} / \bar{R}\right)$, and the alternative $H_{1}$ is that they are different. The two-sample K-S test first calculates the distance between the functions, denoted by $d_{n, \bar{n}}$ and defined by (1).

$$
d_{n, \bar{n}}=\max _{X_{i}}\left|F_{n}\left(X_{i} / R\right)-F_{\bar{n}}\left(X_{i} / \bar{R}\right)\right|
$$

This distance is used in K-S table to find the corresponding pvalue that is the probability of rejecting $H_{0}$ when it is true. The distance $d_{n, \bar{n}}$ and the p-value vary in opposite ways: if $d_{n, \bar{n}}$ is large, the $\mathrm{p}$-value is small ( $H_{0}$ can be rejected with low risk); conversely, if $d_{n, \bar{n}}$ is small, the p-value is large ( $H_{0}$ should not be rejected). In the context of RSA, these indicators serve as criteria for input ranking and factor prioritization. The input are also categorized as “critical”, “important” or "insignificant”, according to the following criterion:

- Critical : $p$-value $<0.01$

- Important : $0.01<p-$ value $<0.1$

- Insignificant : $p$-value $>0.1$

This criterion detects input with 'insignificant' influence and is used for factor fixing.

\subsection{Principle of the proposed traffic rescheduling tool}

The principles presented above have been applied to train traffic rescheduling, in order to better understand the relationships between the inputs and outputs of our model, while accounting for operational constraints.

The rescheduling process is organized in four stages:

1. Problem specification: the decision-maker defines the traffic adjustment variables, their range of variation, the output performance indicator that defines the set of acceptable solutions, and the criteria used to generate Pareto-optimal fronts.

2. Monte Carlo runs: the simulator manager generates the sampling of the input space defined in stage 1 and runs the simulations. Quasi-random sampling, based on Sobol sequences, is used in order to avoid gaps and clusters in the sampled space (Figure 3).

3. Sensitivity analysis: the post-processor generates the $X_{i} / R$ and $X_{i} / \bar{R}$ subsets, computes and plots all cumulative distribution functions, determines the distances and p-values and generates Pareto-optimal fronts.

4. Choice of the rescheduled traffic: the decision-maker interprets the sensitivity analysis results and Paretooptimal front in order to choose the best set of adjustment variables in $X_{i} / R$.

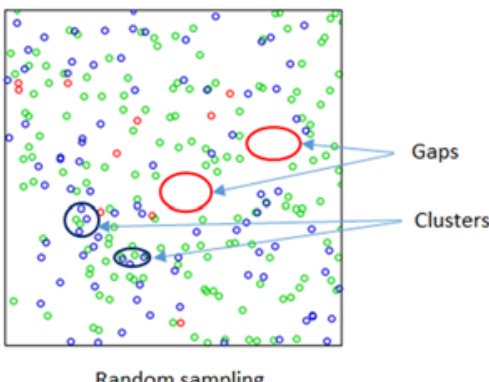

Random sampling

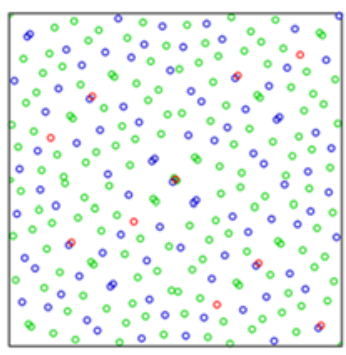

Quasi-Random sampling
Figure 3: Difference between random and quasi-random sampling methods

\section{TEST CASE}

The proposed method has been tested on a simple case representative of a Parisian suburban line (RER D, RoissyPicardie). It is a $35 \mathrm{~km}$ long double-track line, with ten stops and trains travelling in both directions. The line is fed by three $25 \mathrm{kV}$ AC substations, respectively located at 3, 15.8 and 35 $\mathrm{km}$ from the point 0 (REVEST, GONESSE and CREIL substations). Outward trains all leave from the point 0 of the line, but there are three round missions with length of respectively $14 \mathrm{~km}, 20 \mathrm{~km}$ and $35 \mathrm{~km}$. In normal operating conditions, the time interval between train departures is alternatively 5, 6 and 4 minutes. An important constraint to be monitored is the voltage at the train pantographs, which must remain between $19 \mathrm{kV}$ and $29 \mathrm{kV}$ at all times in order to meet the standards and ensure the proper operation of the traction engines.

We suppose a failure of the GONESSE substation. Figure 4 compares the train speed profiles in the nominal case (blue curve) and in the default case (red curve), for 3 trains among the dozens which travel at the same time. The ten stops are visible. Which curve corresponds to which train is not important: what matters is that in the default case, the speed is lower because of power shortage.

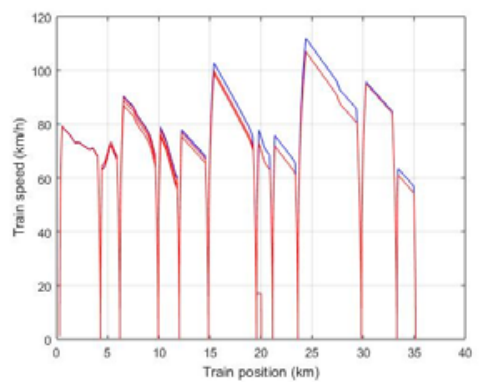

Figure 4: Speed profiles of three trains - blue curves : nominal case - red curves : default case (loss of Gonesse substation).

Figure 5 shows the train pantograph voltage as a function of the train position. One notices the voltage drop, which indicates that the catenary is overloaded in the default case. A lower voltage is not desired because it means higher currents to obtain the same power, more losses, and temperature increase. That is why standards require the voltage to always be above $19 \mathrm{kV}$. This operational constraint is not respected in the default case, and the traffic should be adapted. 


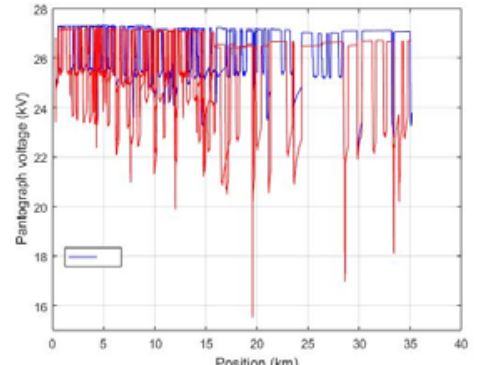

Figure 5: Voltage at the pantograph of three trains - blue curves : nominal case - red curves : default case (loss of Gonesse substation).

To reschedule the traffic, four adjustment variables are considered:

- IDH1 : Increase of the time interval between two successive departures on track1 (leaving Paris)

- IDH2 : Increase of the time interval between two successive departures on track2 (going to Paris)

- $R V 1$ : Speed reduction on track 1 nearby GONESSE substation

- RV2: Speed reduction on track 2 nearby GONESSE substation

The factor mapping setting is done with respect to the catenary voltage, which must remain between $19 \mathrm{kV}$ and $29 \mathrm{kV}$ at all times. Figure 6, Figure 7, Figure 8 and Figure 9 show the cumulative distribution functions corresponding to the four adjustment parameters. Each parameter has a positive impact: increasing those helps to respect the voltage operational constraint.

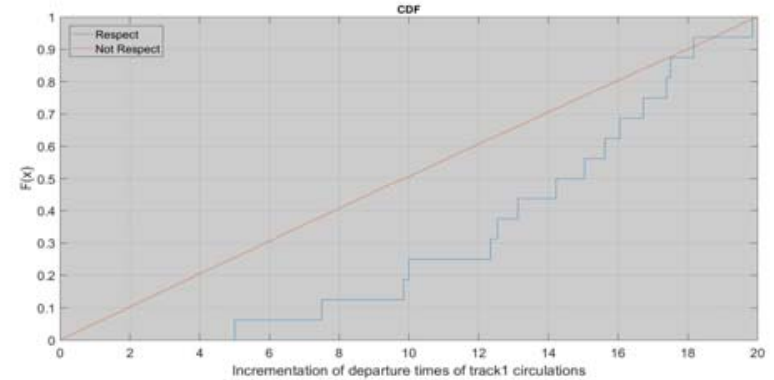

Figure 6: Sensitivity of voltage operationnal constraint to $I \Delta H_{1}$

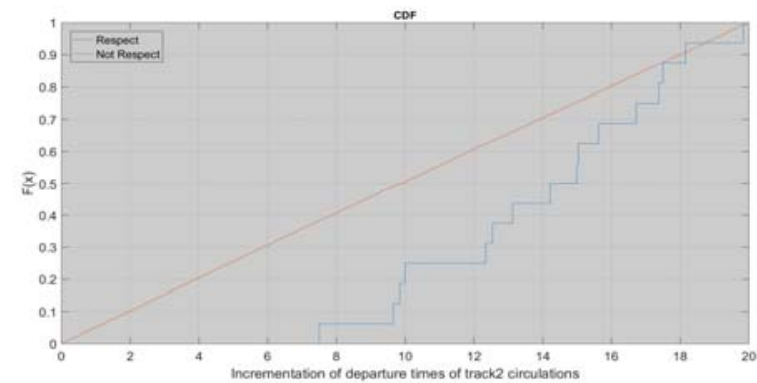

Figure 7: Sensitivity of voltage operationnal constraint to $I \Delta H_{2}$

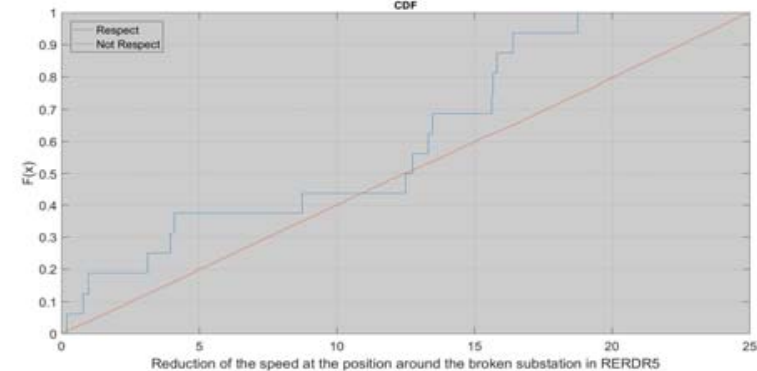

Figure 8: Sensitivity of voltage operationnal constraint to $R V_{1}$

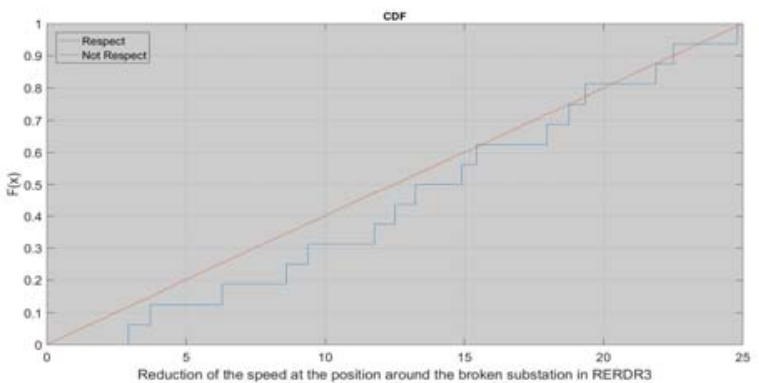

Figure 9: Sensitivity of voltage operationnal constraint to $R V_{2}$

Table 1 presents the results of the two-sample K-S test and quantify the trends observed on the cumulative distribution functions. The adjustment variable $I \Delta H_{1}$ strongly influences positively the results and its range of variation can be reduced to $[5,20] \mathrm{min}$. The adjustment variable $I \Delta H_{2}$ also has a strong positive influences and its range of variation can be reduced to $[7.5,20] \mathrm{min}$. The two adjustment variables $R V_{1}$ and $R V_{2}$ have a weak influence on the output of the voltages. The p-value of $R V_{2}$ is much larger than 0.1 , indicating that its influence is insignificant and that this variable can be dropped.

\begin{tabular}{ccc} 
& $d_{n, \bar{n}}$ & $\mathrm{p}$-value \\
\hline $\boldsymbol{I} \Delta \boldsymbol{H} \mathbf{1}$ & 0.38 & 0.024 \\
$\boldsymbol{I} \boldsymbol{H} \mathbf{2}$ & 0.43 & 0.006 \\
$\boldsymbol{R} \boldsymbol{V} \mathbf{1}$ & 0.2 & 0.15 \\
$\boldsymbol{R} \boldsymbol{V} 2$ & 0.1 & 0.8 \\
\hline
\end{tabular}

Table 1: Results of Two sample Kolmogorov-Smirnov test

These results allow quantifying the influence of each of four adjustment variables on the quality of the tension at the pantographs. It is concluded that the second adjustment variable has the strongest influence on the results, and the variable $R V_{1}$ is insignificant. A set of feasible solutions is produced according to the pantograph tension constraint. It is then possible to extract optimal solutions according to the different criteria defined by the operator.

Figure 10 shows a Pareto plot, which represents the tradeoff between three objective functions: maximizing the network capacity / minimizing the train speed reductions / minimizing the train travel time. Each red point (compromised solution) corresponds to a scenario of adjustments as the Figure 10 shows. 


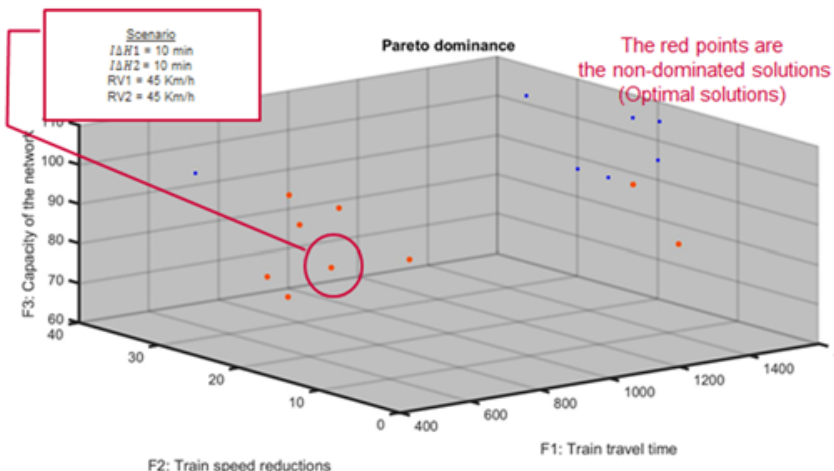

Figure 10: Pareto dominance of three criteria: F1, F2 and F3. The red points are the non-dominated solutions and the blue points are dominated solutions Each point corresponds to a set of adjustments values.

Table 2 shows some efficient solutions that respect the constraints on the outputs of the model. As the incident assumed in this test is serious, it is not possible to have solutions without cancellation of train traffic. Furthermore, as long as the influence of the adjustment variable $R V_{2}$ is insignificant on the results, the operator can leave the initial values of the speeds without reducing them.

\begin{tabular}{lcrcc}
\hline & $\boldsymbol{I} \Delta \boldsymbol{H}_{\mathbf{1}}(\mathbf{m n})$ & $\boldsymbol{I \Delta H}_{\mathbf{2}}(\mathbf{m n})$ & $\boldsymbol{R} \boldsymbol{V}_{\mathbf{1}}(\boldsymbol{k m} / \mathbf{h})$ & $\boldsymbol{R} \boldsymbol{V}_{\mathbf{2}}(\boldsymbol{k m} / \mathbf{h})$ \\
\hline Solution 1 & 12.3 & 12.3 & 14.7 & 89 \\
Solution 2 & 14.2 & 14.2 & 57 & 13.4 \\
Solution 3 & 9.8 & 9.8 & 3.5 & 55.5 \\
Solution 4 & 15 & 15 & 14.2 & 53.6 \\
Solution 5 & 16 & 9.6 & 31.5 & 47.6 \\
\hline
\end{tabular}

Table 2: A set of efficient solutions

Figure 11 shows the train pantograph voltage as a function of the train position for the nominal case (blue curve) and for the default case with rescheduled traffic, according to one of Pareto-optimal points (red curve).

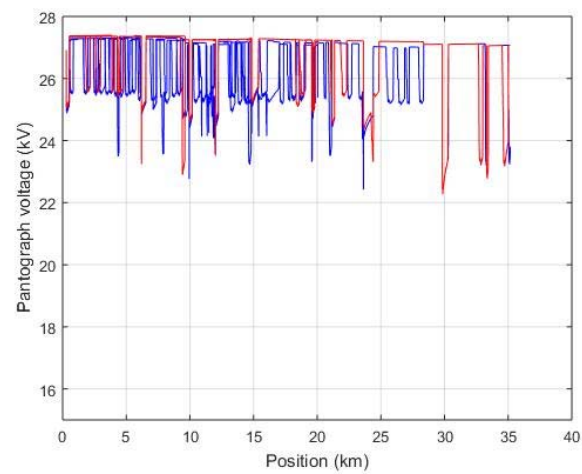

Figure 11: Voltage at the pantograph of three trains - blue curves : nominal case - red curves : default case with rescheduled traffic.

More implementation details and results will be provided in the full paper.

\section{CONCLUSIONS}

In this article a new approach is proposed to reschedule train traffic in the case of electrical infrastructure default. Regional sensitivity analysis is used to account for operational constraints, with a combination of qualitative and quantitative analyses thanks to Kolgorov-Smirnov test. This approach provides useful information such as the influence (positive or negative), the significance and the ranking of the different adjustment variables. It will help the decision-maker to choose an optimal solution according to various criteria.

The test case illustrated in this paper shows that the method used is helpful and accurate in guiding the operators for reorganization the railway traffic. This tool provides a meaningful set of efficient solutions, called Pareto-optimal front, which represents the tradeoff among several criteria. This allows the operator to choose one final solution from this set.

\section{REFERENCES}

[1] J. Krasemann, «Greedy algorithm for railway traffic re-scheduling during disturbances: a swedish case », Progress In IET Intelligent Transport Systems, 2010.

[2] S. Dundar et I. Sahin, « Train rescheduling with genetic algorithms and artificial neural networks for single-track railways ", in Transportation Research Part C, p. 1-15, 2013.

[3] S. Zhan, L. Kroon, L. Veelenturf, et J. Wagenaar « Real-time-high-speed train rescheduling in case of a complete blockage», Progress in Transportation Research Part B, 2016.

[4] I. Louwerse, et D. Huisman « Adjusting a railway timetable in case of partial or a complete blockades», Progress in European journal of operational research, p. 583-593, 2014.

[5] N. Ghaemi, R. Goverde et O. Cats « Railway disruption timetable: Short-turnings in case of complete blockage», in IEEE International conference on intelligent rail transportation (ICIRT), 2016.

[6] S. Binder, Y. Maknoon et M. Bierlaire « Efficient exploration of the multiple objectives of the railway timetable rescheduling problem», in $17^{\text {th }}$ swiss Transport Research Conference, Monte Verita / Ascona, 2016.

[7] B. Desjouis, G. Remy, F. Ossart, C. Marchand, J. Bigeon, E. Sourdille «A new generic problem formulation dedicated to electrified railway systems ", in International Conference on Electrical Systems for Aircraft, Railway, Ship Propulsion and Road Vehicles (IEEE ESRAS 2015), Aachen, Germany, 03-05 March, 2015.

[8] R.T. Celemen, « Making hard decisions: An introduction to Decision Analysis », II Edition, Duxbury Press, Pacific Grove, Calif, USA,ISBN:0534260349.

[9] D. Rios and S. French « A framework for sensitivity analysis in discrete multi-objective decision-making», European Journal of Operational Research 54, 176-190, North-Holland, 1991.

[10] S.L. Avila, A.C. Lisboa, L.krahenbuhl, W.P.Carpes, Jr.,J.A. Vasconcelos, R.R. Saldanha, and R.H.C. Takahashi " Sensitivity Analysis Applied to Decision Making in Multiobjective Evolutionary Optimization», IEEE Transactions on magnetics, 42, april, 2006.

[11] M. Makowski «Multi-objective Decision Support Including Sensitivity Analysis», International Institute for applied systems analysis, Laxenburg,A-2361, Austria.

[12] E. Triantaphyllou and A. Sanchez «A sensitivity analysis approach for some deterministic multi-criteria decision-making methods», Decision Sciences, Volume 28, 1997.

[13] A. Saltelli, S. Tarantola, F. Campolongo, et al., Sensitivity analysis in Practice : A guide to assessing scientific models. London, 2004.

[14] A. Saltelli, M. Ratto, T. Campolongo, F. Cariboni, D. Gatelli, M. Saisana, S. Tarantola, Global sensitivity analysis, The Primer, Wiley, 2008.

[15] G.M. Hornberger, R.C. Spear, “An approach to the preliminary analysis of environmental systems”, Journal of Environmental Management (1981) 12,7-18.

[16] M. Ratto, «Monte Carlo filtering and regional sensitivity analysis (RSA)», DYNAWARE Workshop, CEF2011, San Francisco

[17] Z. Wu, X. Yang and X. Sun, «Application of Monte Carlo filtering method in regional sensitivity analysis of AASHTOWare Pavement ME design», Journal of Traffic and Transportation Engineering, 2017, 4(2): 185-197. 